\title{
La participación de las Comunidades Autónomas en la Unión Europea
}

\author{
Santiago González-Varas Ibáñez
}

\author{
Sumario: 1. PLANTEAMIENTO. 2. PARTICIPACIÓN EXTERNA. 3. LA PARTICIPA- \\ CIÓN INTERNA: FASES ASCENDENTES Y DESCENDENTE. 4. PROPUESTA.
}

\section{PLANTEAMIENTO ${ }^{1}$}

El tema de la Unión Europea y las regiones admite diversas perspectivas. Una de ellas, de creciente actualidad, es la relativa a la participación de las Comunidades Autónomas en el marco institucional europeo.

${ }^{1}$ Sobre el tema que nos ocupa interesa aunque sólo sea reseñar estas disposiciones, reiterando o
completando las citadas en este trabajo:

1. Real Decreto 2105/96, de 21 de septiembre, por el que se crea la Consejería de Asuntos Autonómicos en la Representación Permanente de España (BOE 229, de 21 de septiembre, p. 38934).

2. Real Decreto 1317/97, de 1 de agosto, del Ministerio de Presidencia sobre Comunicación previa a la Administración General del Estado y publicación oficial de los Convenios de cooperación transfronteriza de Comunidades Autónomas y entidades locales con entidades territoriales extranjeras. (BOE 207, de 29 de agosto, p. 25908). Desarrollado por Resolución de 14 de noviembre de 1997 de la Secretaría de Estado para Administraciones Territoriales.

3. Ley 23/1998, de 7 de julio, que regula la cooperación internacional para el desarrollo (BOE 162, de 8 de julio, p. 22755).

4. Reglamento Interno de la CARCE (aprobado en la reunión del 5 de junio de 1997). Deroga el Reglamento interno acordado en la reunión del 14 de junio de 1994.

5. Acuerdo modificando el Acuerdo de Institucionalización de la CARCE, ampliando su ámbito temático (CARCE, 14 de junio de 1994).

6. Acuerdo de institucionalización de la CARCE (CARCE, 29 de octubre de 1992).

Igualmente, interesan los siguientes Acuerdos de la CARCE:

1. Para regular la intervención de las Comunidades Autónomas en las actuaciones del Estado en procedimientos precontenciosos de la Comisión de las comunidades Europeas y en los asuntos relacionados con el Tribunal de Justicia que afecten a sus competencias (CARCE, 29 de noviembre de 1990).

2. En materia de ayudas públicas (CARCE, 29 de noviembre de 1990).

3. Sobre la participación interna de las Comunidades Autónomas en los Asuntos Comunitarios a través de las Conferencias Sectoriales (CARCE, 30 de noviembre de 1994).

4. Sobre la creación de un Consejero para Asuntos Autonómicos en la Representación Permanente de España ante la UE (CARCE, 22 de julio de 1996). 
Para abordar esta cuestión puede hablarse de la existencia de dos modos posibles de participación:

El primero es la participación externa, es decir la participación directa de las CCAA en las instituciones europeas.

El segundo es la participación interna, es decir la participación de las CCAA en los procesos europeos de carácter normativo en los que, en principio, sólo procede la participación directa del Estado como entidad que directamente entabla relaciones jurídicas con las instituciones comunitarias. A su vez, esta variante admite dos modalidades:

La primera se refiere a la fase ascendente de la elaboración y aprobación de normas comunitarias. Desde este punto de vista se trata de averiguar si es posible, o en qué grado o modo, una participación de las Comunidades Autónomas en esta fase.

La segunda alude a la aplicación y ejecución de estas normas una vez han sido aprobadas. Se trata, pues, de una fase propiamente descendente o, cuando menos, así suele denominarse.

5. Relativo al procedimiento para cumplir lo establecido en el Real Decreto 1317/1997, de 1 de agosto, sobre comunicación previa a la Administración General del Estado y publicación oficial de los Convenios de Cooperación Transfronteriza con entidades territoriales extranjeras (CARCE, 2 de diciembre de 1996). Puede consultarse el Real Decreto 1317/1997.

6. Relativo a la participación de las Comunidades Autónomas en los procedimientos ante el Tribunal de Justicia de las Comunidades Europeas (CARCE, 11 de diciembre de 1997).

7. Acuerdo para la participación de las CCAA en los Comités intergubernamentales de control de la Comisión.

8. Sobre Seguridad Social y asistencia sanitaria de las personas que prestan sus servicios en las Delegaciones y Oficinas de las Comunidades Autónomas en Bruselas (CARCE, 11 de diciembre de 1997).

Asimismo, interesan las siguientes disposiciones del Comité de las Regiones:

1. Dictamen «Legislar mejor 1999: Una responsabilidad que debe compartirse».

2. Dictamen «La aplicación de la legislación de la UE por las regiones y los entes locales».

3. Dictamen «El gobierno local y regional en el corazón de Europa».

4. Dictamen «La Conferencia Intergubernamental del 2000».

5. Proceso de elaboración de una Carta de Derechos Fundamentales para la Unión Europea.

6. Reglamento Interno del Comité de las Regiones. 


\section{PARTICIPACIÓN EXTERNA}

En cuanto a la participación externa de las regiones es evidente el límite que impone la Constitución en su artículo 149.1.1 cuando reserva al Estado la materia internacional. No obstante, es preciso conocer la acción «internacional» que desarrollan las CCAA a través del Comité de las Regiones (artículos 263 a 265) y de las Oficinas de las CCAA en Bruselas (apoyadas por el TC: STC 165/1994, de 26 de mayo).

En este contexto debe citarse el Acuerdo de la CARCE (CARCE, 22 de julio de 1996).

Otra vertiente, dentro de esta misma participación externa, es aquélla que lleva a discutir la posibilidad de una participación de las CCAA en el Consejo, la Comisión y el TJCE.

El propio TCE (artículo 203) prevé la posibilidad de los «ministros regionales» en el Consejo y, en el caso español, se han adoptado algunos acuerdos por el Congreso o el Senado (o la propia CARCE) que reafirman esta posibilidad sin que hasta el momento se haya llegado a algún resultado.

En la Comisión Europea un «Acuerdo (de la CARCE) para la participación de las CCAA en los Comités intergubernamentales de control de la Comisión» ha dado pie a la participación de las CCAA en numerosos Comités de control, aunque tampoco se ha institucionalizado un cauce de participación.

En el TJCE existe igualmente un Acuerdo (de la CARCE) (CARCE, 11 de diciembre de 1997) que procura un sistema de defensa unitaria entre Estado y CA cuando existen intereses comunes.

No acaban con esto los mecanismos de participación a favor de las CCAA. Es preciso citar las conferencias de las regiones europeas con competencias legislativas. Son concretamente la Conferencia de Asambleas Legislativas Regionales Europeas (CALRE) en 1997, y, por otra parte, la Conferencia de Presidentes de Regiones con Poderes Legislativos (REG LEG) en 2000. La CALRE ha venido celebrando desde su constitución en 1997 reuniones anuales que se cierran con una Declaración en la que se recogen las principales pretensiones y aspiraciones de las regiones parlamentarias en la Unión Europea.

Por su parte, la Conferencia de Presidentes de Regiones con Poderes Legislativos (REG-LEG) celebró en noviembre de 2003 su IV edición en 
Salzburgo. Esta IV Conferencia concluyó con una Declaración en favor de la aprobación de la Constitución Europea, aunque también se solicitó en la misma una mayor implicación de estas regiones de acuerdo con sus responsabilidades.

También es preciso considerar la cooperación transfronteriza como competencia autonómica que permite una importante acción internacional a las CCAA. Los acuerdos de cooperación transfronteriza permiten celebrar (a las Comunidades Autónomas o a sus municipios) convenios con las autoridades competentes de otros Estados (en nuestro caso Francia o Portugal).

Hemos de citar el famoso Convenio Marco de 5.2.1980 sobre cooperación transfronteriza. Este Convenio sugería la posibilidad de celebrar Acuerdos interestatales.

De ahí que España, al igual que otros Estados, considerara insuficiente este Convenio Marco y por eso aprobara con Francia un Acuerdo específico, igualmente conocido: el Tratado de Bayona. En el Norte de España rige este Tratado desde el 10.3.1995, entrando en vigor en 1997, según el cual se permiten este tipo de acuerdos transfronterizos.

Para la eficacia de estos acuerdos transfronterizos con un Tratado interestatal de cobertura basta con la previa comunicación del proyecto de acuerdo transfronterizo a la Administración del Estado en virtud del Real Decreto 1317/1997. Finalmente, puede considerarse un Protocolo de 1996, no ratificado por España.

\section{LA PARTICIPACIÓN INTERNA: FASES ASCENDENTE Y DESCENDENTE}

Esta participación se realiza a través de las conferencias sectoriales y las conferencias bilaterales.

Una primera referencia sobre las citadas conferencias puede encontrarse, como es sabido, en la Ley 30/1992, de 26 de noviembre, de Régimen Jurídico de las Administraciones Públicas y del Procedimiento Administrativo Común.

Su artículo 5 afirma que «la Administración General del Estado y la Administración de las Comunidades Autónomas pueden crear órganos para la cooperación entre ambas, de composición bilateral o multilateral, de ámbito general o de ámbito sectorial, en aquellas materias en las que 
exista interrelación competencial, y con funciones de coordinación o cooperación según los casos» ${ }^{2}$.

En el tema que nos ocupa interesa la Conferencia para Asuntos relacionados con las Comunidades Europeas (CARCE).

Esta Conferencia, constituida en 1988, fue institucionalizada en 1992 mediante un Acuerdo de institucionalización de la Conferencia para Asuntos relacionados con las Comunidades Europeas (CARCE). Su objetivo principal es desarrollar esquemas de participación de las CCAA en ambas fases ascendente y descendente referidas anteriormente.

Su propio funcionamiento puso de manifiesto la necesidad de acometer una regulación normativa para consolidar este sistema y garantizar un procedimiento de intervención efectiva de las CCAA en la elaboración y ejecución del Derecho comunitario. De ahí que se dictara la Ley 2/1997, de 13 de marzo, que regula la Conferencia para Asuntos relacionados con las CCEE y posteriormente el Reglamento interno (que deroga el Reglamento anterior de 14 de junio de 1994 que se había aprobado conforme al propio Acuerdo de institucionalización de 1992).

\footnotetext{
2 Dice el artículo: A efectos de lo establecido en el presente Capítulo, no tienen la naturaleza de órganos de cooperación aquellos órganos colegiados creados por la Administración General del Estado para el ejercicio de sus competencias en cuya composición se prevea que participen representantes de la Administración de las Comunidades Autónomas con la finalidad de consulta. 2. Los órganos de cooperación de composición bilateral y de ámbito general que reúnan a miembros del Gobierno, en representación de la Administración General del Estado, y a miembros del Consejo de Gobierno, en representación de la Administración de la respectiva Comunidad Autónoma, se denominan Comisiones Bilaterales de Cooperación. Su creación se efectúa mediante acuerdo, que determina los elementos esenciales de su régimen. 3. Los órganos de cooperación de composición multilateral y de ámbito sectorial que reúnen a miembros del Gobierno, en representación de la Administración General del Estado, y a miembros de los Consejos de Gobierno, en representación de las Administraciones de las Comunidades Autónomas, se denominan Conferencias Sectoriales. El régimen de cada Conferencia Sectorial es el establecido en el correspondiente acuerdo de institucionalización y en su reglamento interno. 4. La convocatoria de la Conferencia se realizará por el Ministro o Ministros que tengan competencias sobre la materia que vaya a ser objeto de la Conferencia Sectorial. La convocatoria se hará con antelación suficiente y se acompanará del orden del día y, en su caso, de la documentación precisa para la preparación previa de la Conferencia. 5. Los acuerdos que se adopten en una Conferencia Sectorial se firmarán por el Ministro o Ministros competentes y por los titulares de los órganos de gobierno correspondientes de las Comunidades Autónomas. En su caso, estos acuerdos podrán formalizarse bajo la denominación de Convenio de Conferencia Sectorial. 6. Las Conferencias Sectoriales podrán acordar la creación de comisiones y grupos de trabajo para la preparación, estudio y desarrollo de cuestiones concretas propias del ámbito material de cada una de ellas. 7. Con la misma finalidad, y en ámbitos materiales específicos, la Administración General del Estado y las Administraciones de las Comunidades Autónomas podrán constituir otros órganos de cooperación que reúnan a responsables de la materia. 8. Cuando la materia del ámbito sectorial de un órgano de cooperación de composición multilateral afecte o se refiera a competencias de las Entidades Locales, el pleno del mismo puede acordar que la asociación de éstas de ámbito estatal con mayor implantación sea invitada a asistir a sus reuniones, con carácter permanente o según el orden del día.
} 
El esquema de la Ley es el siguiente:

Artículo 1: definición.

Artículo 2: composición

Artículo 3: funciones.

Artículo 4: régimen de funcionamiento.

Disposiciones Adicionales: Cooperación bilateral y Ceuta y Melilla.

Conforme a esta Ley, «La Conferencia para Asuntos Relacionados con las Comunidades Europeas, que se regula en la presente Ley, es un órgano de cooperación entre el Estado y las Comunidades Autónomas para articular adecuadamente la concurrencia de éstas en las cuestiones propias de su participación en los asuntos comunitarios europeos. En particular, la Conferencia debe garantizar la participación efectiva de las Comunidades Autónomas en la fase de formación de la voluntad del Estado ante las instituciones comunitarias y en la ejecución del Derecho comunitario» ${ }^{3}$.

${ }^{3}$ En cuanto a su composición, la Conferencia estará constituida por el Ministro de Administraciones Públicas, que la presidirá, y por el Consejero que, como responsable de los asuntos que integran el ámbito de materias de la misma, sea designado por cada Comunidad Autónoma, de acuerdo con sus normas de organización interna. En la representación de la Administración del Estado se integrarán tanto el Secretario de Estado de Política Exterior y para la Unión Europea como el Secretario de Estado para las Administraciones Territoriales.

Y respecto de sus funciones, la Conferencia, como órgano de cooperación, de consulta y deliberación entre el Estado y las Comunidades Autónomas, y sin perjuicio de sus respectivas facultades de actuación en el marco de sus competencias, entenderá de las siguientes materias:

— La información a las Comunidades Autónomas y la discusión en común sobre el desarrollo del proceso de construcción europea.

- La articulación de mecanismos para hacer efectiva la participación de las Comunidades Autónomas en la formación de la voluntad del Estado en el seno de las Comunidades Europeas.

- El tratamiento y resolución con arreglo al principio de cooperación de aquellas cuestiones de alcance general o contenido institucional relacionadas con las Comunidades Europeas como las siguientes:

- Procedimientos técnicos para asegurar la recepción de la información comunitaria de carácter general por parte de las Comunidades Autónomas.

- Técnica normativa tanto para incorporar las directivas al Derecho interno como para aplicar, desarrollar o ejecutar reglamentos y decisiones.

- Fórmulas de participación en los procedimientos internos para el cumplimiento de obligaciones ante las instituciones comunitarias.

- Problemas planteados en la ejecución del Derecho comunitario por implicar a varias políticas comunitarias o exigir medidas internas con un cierto grado de coordinación temporal o materi- 
Sobre el tema que nos ocupa, de la participación que en concreto corresponde a las CCAA en las referidas fases ascendente y descendente, hay que estar a la Resolución de 10 de marzo de 1995 de la Secretaría de Estado para Administraciones Territoriales que publica el Acuerdo de la CARCE de 30 de noviembre de 1994 sobre la participación interna de las Comunidades Autónomas en los Asuntos Comunitarios a través de las Conferencias Sectoriales (publicada en el BOE 69, de 22 de marzo, p. 9037 y 78 , de 1 de abril, p. 10045).

En este Acuerdo primero se establecen unos principios generales o procedimiento marco en el que se determina el contenido necesario de la participación de las Comunidades Autónomas, tanto en lo que respecta a la fase ascendente como en lo relativo a la fase descendente.

En este sentido, «en el desarrollo por cada Conferencia Sectorial del procedimiento marco deberá ser tenida en cuenta, a efectos de determinar el grado de intensidad y el contenido concreto de la participación de las Comunidades Autónomas, la naturaleza y el nivel de competencias, tanto de las asumidas por las Comunidades Autónomas como de las reservadas al Estado».

Los criterios son los siguientes:

1. Cuando el asunto comunitario europeo afecte exclusivamente a las competencias reservadas del Estado y las Comunidades Autónomas invoquen su interés, la Administración del Estado les informará oportunamente en el marco de la Conferencia Sectorial respectiva.

2. Cuando los aspectos esenciales de un asunto comunitario afecten a las competencias legislativas exclusivas de las Comunidades Autónomas, si en el procedimiento de concertación interno, previo a la decisión del Consejo, se ha llegado a una posición común entre ellas, ésta será tenida

- Cuestiones relativas a la participación de las Comunidades Autónomas en los asuntos relacionados con las Comunidades Europeas que carezcan de una Conferencia Sectorial o instrumento equivalente donde ser tratadas.

- El impulso y seguimiento del procedimiento de participación de las Comunidades Autónomas, a través de las respectivas Conferencias Sectoriales u organismo equivalente, en las políticas o acciones comunitarias que afectan a las competencias de aquéllas.

- Garantizar el cumplimiento en las Conferencias Sectoriales de los procedimientos y fórmulas de participación de las Comunidades Autónomas previstos en las materias 3.c) y 4), disponiendo la adecuada aplicación de los mismos.

- El tratamiento de aquellas otras cuestiones de la participación de las Comunidades Autónomas en los asuntos relacionados con las Comunidades Europeas que estimen oportuno. 
en cuenta de forma determinante a efectos de fijar la posición negociadora inicial del Estado.

3. En aquellos asuntos que incidan sobre competencias compartidas o concurrentes del Estado y de las Comunidades Autónomas, en los que previamente a la decisión del Consejo exista un acuerdo entre la posición común de las Comunidades Autónomas y la posición de la Administración del Estado, tal acuerdo será determinante a efectos de fijar la posición negociadora inicial del Estado.

4. En los supuestos 2 y 3 , si la posición inicial del Estado experimentase variación sustancial como consecuencia del proceso de negociación comunitaria, siempre que los plazos lo permitan, la Administración del Estado, en el seno de la respectiva Conferencia Sectorial, informará a las Comunidades Autónomas sobre la modificación para facilitar una nueva posición común de éstas y, en su caso, su acuerdo con la posición de la Administración del Estado. Cuando los plazos no lo permitan, la Administración del Estado, a solicitud de las Comunidades Autónomas, explicará los motivos de que la posición expresada por el Estado en el seno del Consejo haya variado respecto de la inicialmente acordada.

5. En el supuesto de que las decisiones sean susceptibles de originar aumento de gasto o disminución de ingresos de las Administraciones Públicas, la posición común de las Comunidades Autónomas se consensuará con la posición de la Administración del Estado.

6. En aquellos asuntos en los que no se hubiese alcanzado una posición común por parte de las Comunidades Autónomas, la Administración del Estado tomará conocimiento de los argumentos expresados por las Comunidades Autónomas.

En cuanto a la participación de las Comunidades Autónomas en la fase de formación de la voluntad del Estado (fase ascendente) la participación de la Comunidades Autónomas en la fase de formación de la voluntad del Estado tendrá como contenido necesario el que a continuación se establece:

1. La Conferencia Sectorial remitirá sin dilación a las Comunidades Autónomas la propuesta de la Comisión fijando, en función de los plazos de tramitación en el Consejo, un término para expresar su postura.

2. De conformidad con el sistema que acuerde cada Conferencia Sectorial o, en su defecto, siempre que lo solicite al menos una Comunidad Autónoma, la propuesta de la Comisión deberá incluirse en el orden del día del órgano especializado de la Conferencia -comisión, grupo de trabajo, o reunión ad 
hoc-a efectos de su deliberación y consideración conjunta entre la Administración del Estado y las Administraciones de las Comunidades Autónomas.

3. La Conferencia Sectorial informará regularmente a las Comunidades Autónomas de la evolución de la propuesta de la Comisión en el seno del Consejo.

4. En los procedimientos de cooperación y codecisión, las Comunidades Autónomas serán informadas de todas las sucesivas modificaciones que se produzcan a lo largo del proceso de adopción de la iniciativa.

En el caso de convocatoria del Comité de Conciliación previsto en el procedimiento de codecisión se informará a las Comunidades Autónomas sobre sus deliberaciones.

5. La Conferencia Sectorial remitirá sin dilación a las Comunidades Autónomas el texto de la propuesta de la Comisión que se incluya en el orden del día del Consejo.

6. La Conferencia Sectorial remitirá sin dilación a las Comunidades Autónomas el orden del día provisional de la sesión del Consejo. Teniendo en cuenta el contenido del orden del día y el resultado del procedimiento de participación desarrollado, cada Conferencia Sectorial valorará la oportunidad de una reunión del Pleno de la misma antes de la sesión del Consejo.

En cuanto a la participación de las Comunidades Autónomas en la fase descendente de aplicación del Derecho comunitario europeo y de los actos de las instituciones es preciso partir del principio de autonomía institucional que rige en todos los Estados miembros en lo relativo al cumplimiento de las obligaciones derivadas de los Tratados, así como del principio de que la aplicación en España del Derecho comunitario europeo debe llevarse a cabo conforme a la distribución de competencias resultante del bloque de constitucionalidad, la Administración del Estado y las Administraciones de las Comunidades Autónomas.

La participación en la fase descendente implica, como contenido necesario, los siguientes compromisos relativos a la aplicación del Derecho comunitario europeo y de los actos de las instituciones.

1. Cuando la aplicación consista en la aprobación de normas.

1.1. Las Administraciones que proyecten la aprobación de una norma, bien para desarrollar o completar un reglamento o decisión comunitario, 
bien para efectuar la transposición de una directiva comunitaria, pondrán en conocimiento de la Conferencia Sectorial el texto del proyecto.

1.2. Cuando en la Conferencia Sectorial la Administración del Estado y las Administraciones de las Comunidades Autónomas coincidan en la necesidad de dar un contenido semejante o equivalente al proceso normativo interno derivado de la aplicación del Derecho comunitario, la cuestión será incluida en el orden del día del correspondiente órgano especializado de la Conferencia Sectorial al objeto de elaborar una propuesta de acuerdo y elevarla al Pleno de la Conferencia.

2. Cuando la aplicación consista en la ejecución de actuaciones administrativas.

2.1. La Administración del Estado y las Administraciones de las Comunidades Autónomas se informarán regularmente, a través de la Conferencia Sectorial, de aquellas actuaciones administrativas en proyecto o en curso que se deriven de la aplicación del Derecho comunitario.

2.2. Cuando en la Conferencia Sectorial la Administración del Estado o las Administraciones de las Comunidades Autónomas propongan la necesidad de dar un contenido semejante o equivalente al proceso de ejecución administrativa en aplicación del Derecho comunitario, la cuestión será incluida en el orden del día del correspondiente órgano especializado de la Conferencia Sectorial al objeto de elaborar una propuesta de acuerdo y elevarla al Pleno de la Conferencia.

3. Cuando la aplicación consista en el desarrollo de programas comunitarios.

3.1. La Administración del Estado informará regularmente a las Administraciones de las Comunidades Autónomas, a través de la Conferencia Sectorial, de aquellos programas comunitarios que gestione, abiertos o no a la participación de las Comunidades Autónomas.

3.2. Las Administraciones de las Comunidades Autónomas informarán regularmente a la Administración del Estado de su participación en aquellos programas comunitarios que no sean coordinados por la Administración del Estado.

Junto a los mecanismos referidos anteriormente es preciso conocer también las Conferencias Bilaterales. Así, en la disposición adicional primera de la Ley reguladora de la CARCE se prevé la «Cooperación bilateral»: «aquellas cuestiones propias de la participación en los asuntos relacionados con las 
Comunidades Europeas, que afecten en exclusiva a una Comunidad Autónoma o que tengan para ésta una vertiente singular en función de su especificidad autonómica, se tratarán, a iniciativa de cualquiera de las partes y de mutuo acuerdo, mediante instrumentos de cooperación de carácter bilateral».

En la práctica estas conferencias bilaterales no se han desarrollado, ya que es lógico que la participación se encauce a través de los otros mecanismos generales previstos en el marco de la cooperación general entre Estado y CCAA.

Como complemento de las afirmaciones precedentes pueden reseñarse ciertas noticias de actualidad sobre el tema que nos ha ocupado, tomando como fuente los periódicos de los últimos días (Razón Digital del viernes 10 de diciembre de 2004; Europa Sur domingo, 12 de diciembre de 2004, Europa Press 8 Dic.).

Las Comunidades estarán presentes en cuatro Consejos de Ministros de la UE mediante representantes elegidos en las Conferencias Sectoriales. Cada representante permanecerá al menos un semestre. Todas las CCAA tendrán ocasión de tener un representante. Las Comunidades estarán representadas en los Consejos de Empleo, Política Social, Sanidad y Consumidores, que incluye Turismo; Agricultura y Pesca; Medio Ambiente y Educación, Juventud y Cultura. En concreto, un consejero autonómico, elegido de forma rotatoria, representará a todas las autonomías en cuatro Consejos de Ministros de la Unión Europea, en función de las propuestas que se aborden en la reunión de la Conferencia de Asuntos Relacionados con las Comunidades Europeas (CARCE) que se celebrará en el Senado.

Por otra parte, Galicia y Andalucía han sido las primeras en designar a sus representantes comunitarios en la Representación Permanente de España ante la Unión Europea, la REPER. El número de representantes podría ampliarse.

Estos Acuerdos son fruto de la Conferencia para Asuntos Relacionados con las Comunidades Europeas (CARCE).

\section{PROPUESTA ${ }^{4}$}

Lege ferenda cabe profundizar en la posibilidad de una política regional comunitaria. Suele entenderse por política regional comunitaria temas

${ }^{4}$ Las siguientes afirmaciones se basan en, y completan lo contenido en, mi libro España no es diferente, Editorial Tecnos, Madrid 2000 y en el trabajo «Una mayor europeización de la política regio- 
tales como la participación de las Regiones en el ejercicio de competencias de la Comunidad Europea, el Comité de las Regiones, etc. A estas cuestiones se acaba de hacer referencia supra en este trabajo.

Ahora bien, si Europa aspira, como parece, a ser una realidad más próxima a un «Estado» y elabora al efecto una Constitución, y perfecciona su sistema de competencias, y desea una mayor integración no sólo económica sino también política, no parece descabellado que Europa asuma una cierta influencia, aunque mínima, en el proceso de definición del papel de las regiones, sus regiones, en Europa.

Si se pensara en «clave europea», y conociendo la historia del viejo continente, puede plantearse el debate de unos posibles marcos futuros de participación de las regiones desde un punto de vista europeo y europeísta. No se legitima que los Estados lleguen a agotar por completo la definición del papel que ha de corresponder a sus regiones.

Es cierto que el punto de partida está en el principio de autonomía institucional, en el de subsidiariedad y en el respeto de los Estados (la Constitución Europea lo recuerda oportunamente en su artículo I-5,1).

Pero también ha de reconocerse que ciertos Estados europeos tienen tradiciones jurídicas y políticas similares entre sí (me refiero en concreto a Alemania, España, Francia, Italia y el Reino Unido como Estados compuestos por esencia).

Es evidente que una plena igualación de todas las regiones no es viable a nivel europeo, porque la perspectiva nacional (es decir, la definición en clave nacional del papel que en cada Estado ha de corresponder a sus regiones) sigue y tiene que seguir primando o dominando. Ahora bien, habría un mínimo de influencia de la Unión europea, más allá de los cauces institucionales del Comité de las Regiones o perspectivas análogas. Se trata, más bien, de que la Unión Europea pueda repercutir en cierta medida en la definición del papel que ha de corresponder a las regiones europeas. Esta propuesta se traduciría en la definición de unos marcos (aunque generales) de mínimos y máximos competenciales a favor de las regiones.

Un caso llamativo, desde una perspectiva europea, lo presentan algunas regiones que están divididas por fronteras nacionales y, mientras que en

nal y la necesidad de una Ley de normalización del español como consecuencia», Revista de Administración y función pública de Castilla y León, n. ${ }^{\circ} 1$, 2004. Igualmente, "Spain is different hoy», Diario $A B C$, Sección Tribuna (17 de abril de 2002, p. 71) y «¿Europa de las regiones?», Diario $A B C$, Sección Tribuna, 27 de septiembre de 2002 p. 61. 
unos Estados (España) las regiones (Cataluña y el País Vasco) gozan de unas enormes prerrogativas (competenciales, de soberanía, lingüísticas, políticas, etc.) del otro lado de la frontera las mismas regiones no tienen siquiera reconocida una entidad regional por ser una provincia o departamento que forma parte de una región más amplia (por ejemplo, de la Aquitania). ¿Cómo explicar estas anomalías en clave europea? Insistiendo en la perspectiva que abre el «pensamiento europeísta», parece lógico afirmar que si las regiones españolas a las que acabo de referirme hubieran estado del otro lado de la frontera, sólo existiría en ellas una cultura francesa y una uniformidad política y lingüística basada en el hecho nacional francés.

Los grandes Estados europeos han tenido una tradición similar y son parte de una misma familia europea. En los distintos Estados que he mencionado (Alemania, Francia, España, Italia y el reino Unido) existen ciertas regiones que representan el núcleo (también históricamente) del Estado (Inglaterra en el Reino Unido; la zona central vinculada al Monarca en París en Francia, Prusia en Alemania, Castilla y Aragón en España) y otras zonas que, sin perjuicio de haber aportado a la realización del Estado donde se integran, tienen una cierta dimensión especial (Escocia y Gales en el Reino Unido; País Vasco francés, Alsacia, Córcega, Bretaña en Francia, etc.; Cerdeña, Sicilia, Lombardía etc. en Italia; Baviera, Hesse, Sajonia, etc. en Alemania; Cataluña, País Vasco español en España) junto a otras zonas o regiones que puedan situarse en una dimensión o escala intermedia.

En el presente estamos en condiciones de identificar claramente estas cuestiones para establecer a nivel europeo unos parámetros competenciales de máximos y mínimos.

Junto a estos Estados mencionados existen otros, en Europa, con una problemática tampoco dispar entre sí. Son los Estados, con identidad nacional en la actualidad, pero desgajados históricamente de los Estados del grupo anteriormente mencionado (los antiguos imperios germánicos, francés, británico y español). Me refiero lógicamente a Austria, Dinamarca, Luxemburgo, Holanda, las dos partes belgas (una francesa, otra germánica), Irlanda y Portugal.

Es ilógica la equiparación que se pretende a veces entre todos los Estados europeos, queriendo implantar una política regional por ejemplo en Portugal o Dinamarca con iguales consecuencia que en Francia o el Italia o Alemania, España y el Reino Unido. Por mucho que se pretenda una regionalización en Portugal o en Dinamarca ésta nunca puede tener el mismo sentido que en aquellos otros Estados. 
Estarían justificadas las diferencias entre los Estados de cada uno de los dos grupos establecidos; pero no los planteamientos radicalmente opuestos entre sí. La Unión Europea tiene un compromiso con las regiones y con los Estados, y de ahí que debiera a aquella en el futuro involucrarse en la definición política de los Estados y de sus regiones. En el primer grupo mencionado de Estados podría fijarse un nivel máximo y mínimo de competencias en el marco de un sistema de descentralización política; no así en el segundo grupo de Estados por razones obvias. Es decir, en Portugal o en Irlanda o Dinamarca falta la característica histórica fundamental presente en aquellos otros Estados a la que ya me he referido. Si es preciso podría consolidarse un tercer grupo de Estados, de carácter residual, que aglutina aquellos de posición intermedia entre aquellos otros dos grupos.

Es preciso reconocer que si este planteamiento europeísta no vence aún hoy en Europa es porque en los grandes Estados la ratio o el interés es principalmente nacional, pese a lo que uno hubiera podido pensar.

En todo caso, es evidente que el caso español debe ponerse en relación con los Estados mencionados (Alemania, Francia, Italia y el Reino Unido) y no con otros diferentes, tal como a veces se hace a mi juicio impropiamente. El caso belga, por ejemplo, no tiene mucho que ver con el nuestro porque, además de las razones expuestas, Bélgica es suma de dos partes de identidad nacional (francesa y germánica). Bélgica no es, como Alemania o Francia o Italia o el Reino Unido o España un Estado auspiciado por un determinado poder territorial de identidad propia que termina extendiéndose a los distintos confines del territorio nacional.

Es más, en España un estudio histórico serio pone de manifiesto que el papel de nuestras regiones nunca ha sido tan destacado como el de otras, tales como Baviera, Sajonia, Escocia, Aquitania o Alsacia. En estos mismos términos debería abordarse el tema lingüístico. No tiene sentido, en clave europea, que el alsaciano o el bretón sean considerados dialectos o lenguas que no pueden emplearse en la vida pública (universitaria, legislativa, comunal, las señales de tráfico, etc.) mientras que nuestras lenguas españolas sean consideradas prácticamente al mismo nivel que la lengua oficial del Estado. España es un Estado o zona de Europa donde se plantean los mismos problemas lingüísticos que en Alemania, Francia, Italia o el Reino Unido.

En la línea de estas afirmaciones «europeístas» se observan ciertas tendencias que manifiestan la posibilidad de un enfoque europeo general del tema regional, tal como estamos propugnando. Se consolida hoy por 
ejemplo un marco de conferencia de las «regiones con competencia legislativa» (y otras que deberían tenerla, me refiero a las regiones francesas que son regiones de igual naturaleza que Escocia, Cerdeña, Baviera o Cataluña). Estrasburgo, por su parte, nos informa de que es posible un sistema de niveles competenciales de máximos y mínimos, a nivel europeo, tal como corrobora la Carta Europea de las lenguas regionales o minoritarias de 16 de agosto de 1998. La técnica seguida, en efecto, por esta Carta es la de plantear un máximo y un mínimo de competencias lingüísticas a favor de las regiones de los Estados miembros. Bien es cierto que, no obstante, los Estados más nacionales como lo son Alemania o Francia se han apresurado a desvirtuar por completo su aplicación práctica.

La europeización de la política regional abre una vía para la solución futura de conflictos entre Estados y regiones, haciendo ver a éstas dónde están y cuáles son sus aspiraciones legítimas y cuáles no.

Para preparar el terreno a una auténtica política europea regional podría empezarse por un sistema de decisiones «orientativas» no vinculantes de la Unión Europea para ir avanzando o un sistema de «cláusulas de salvaguarda» a favor de los Estados. 